\title{
Two-dimensional Navier-Stokes simulation of deformation and break up of liquid patches
}

\author{
Stéphane Zaleski \\ Jie Li \\ Sauro Succi酒 \\ Laboratoire de Modélisation en Mécanique, \\ CNRS URA 229, Université Pierre et Marie Curie (Paris 6), \\ Paris, France
}

(February 22, 1995)

\begin{abstract}
The large deformations and break up of circular 2D liquid patches in a high Reynolds number $(R e=1000)$ gas flow are investigated numerically. The 2D, plane flow Navier-Stokes equations are directly solved with explicit tracking of the interface between the two phases and a new algorithm for surface tension. The numerical method is able to pursue the simulation beyond the breaking or coalescence of droplets. The simulations are able to unveil the intriguing details of the non-linear interplay between the deforming droplets and the vortical structures in the droplet's wake.

02.60.Cb,47.55.Dz
\end{abstract}

Typeset using REVTEX 
The dynamical processes leading to breakup of a single lump of fluid into several pieces and, conversely, the coalescence of several pieces into a single one, have captured considerable attention from both the theoretical [1 3] and experimental side [4 6]. From the theoretical point of view, much of the interest stems from the fact that breakup/coalescence provide eminent examples of topological singularity formation in hydrodynamic systems. Practical interest is even more evident if one thinks of the enormous wealth of physico-chemical phenomena in which drop formation and break up play a crucial role. The merging of galaxies, spray vaporization combustion in diesel engines and deformation of biological cells are just but a few representative examples. In many instances the primary question is just to assess under which conditions breakup/coalescence do occur. However it is clear that the exploration of what happens after breakup/coalescence is of paramount importance to deepen our understanding. The latter question is even more formidable, but the numerical simulations presented in this Letter should offer a preliminary answer.

The physics of drop deformation and break up is governed by the competition between hydrodynamic stresses, viscous or inertial, which act to deform the droplet, and surface tension which opposes increase of the surface area, and thus tends to restore weakly deformed objects to a spherical shape.

In this article we present simulations of droplets of liquid moving about in a gas environment. The mathematical idealisation of this problem is that of a $2 \mathrm{D}$, incompressible Newtonian flow with surface tension on the interface and viscous dissipation in the bulk. The momentum balance equations are the Navier-Stokes equations for an incompressible fluid of variable viscosity

$$
\rho\left(\partial_{t} \mathbf{u}+\mathbf{u} \cdot \nabla \mathbf{u}\right)=-\nabla p+\nabla \cdot(\mu \mathbf{E})+\nabla \cdot\left[\sigma(\mathbf{I}-\mathbf{n} \otimes \mathbf{n}) \delta_{S}\right]
$$

where $E_{i j}=\partial_{i} u_{j}+\partial_{j} u_{i}$ is the rate of strain tensor, $I_{i j}=\delta_{i j}$ the unit tensor, $\delta_{S}$ a distribution concentrated on the interface, $\mathbf{n}$ is the normal to the interface, and $\sigma$ the surface tension coefficient. The fluid density $\rho$ and the viscosity $\mu$ are constant in each phase but vary from phase to phase. The specific form of the surface tension term used is equivalent to the more 
classical Laplace law [7,8]. We consider incompressible flow with $\nabla \cdot \mathbf{u}=0$, and the interface moves at a normal velocity $U_{I}=\mathbf{u} \cdot \mathbf{n}$. In addition to these equations, a condition is needed for the reconnection of interfaces. In a real flow, this reconnection is a complex process, involving long range molecular interactions between interfaces. It is impossible with current computing capabilities to simulate both the large scale, high Reynolds flow around a droplet and the molecular interactions. The pragmatic alternative is to introduce a cutoff scale $\delta_{C}$ below which one will not attempt to model the interface physics. Such a cutoff is consistent with the spontaneous behavior of the Volume of Fluid methods described below, in which liquid sheets of thickness smaller than the mesh size $h$ tend to break.

Taking as a length scale the diameter $D$ of the droplet and a characteristic speed $U$ of the flow, the problem has 4 dimensionless numbers, the gas and liquid Reynolds numbers $R e_{i}=$ $\rho_{i} U D / \mu_{i}, i=L, G$, the gas Weber number $W e_{G}=\rho_{G} U^{2} D / \sigma$ and the density ratio $\rho_{L} / \rho_{G}$. Experiments show that droplets suddenly placed in a high speed flow break when $W e_{G}$ is between 10 and 20 [9,10]. Several droplet break up regimes have been identified [11, [12], and the basic theory involves various instability mechanisms for the liquid gas surface, [13, 14] following the pioneering work of Taylor [15]. The authors are not aware, at this date, of numerical simulations of this problem beyond relatively small droplet deformations. However it must be noted that simulations exist in the limit of vanishing $R e_{i}$ 四.

We used the method described in [8,16]. A first order in time explicit integration of equations (1) was performed using the MAC staggered finite difference grid for the momentum balance equation. The $2 \mathrm{D}$ version of the method was used in order to achieve calculations on larger grids. The incompressibility condition is accurately met by a projection method 17 with the help of a multigrid algorithm [18]. Surface tension is implemented in a momentum conserving way, via the introduction of a non-isotropic stress tensor concentrated near the interface [8]. This representation of surface tension stresses is especially interesting for the simulation of break up, since it avoids the singularity which would occur in the continuum limit when interfaces change topology and the curvature becomes locally infinite.

The velocity field $\mathbf{u}$ obtained at each time step is used to propagate the interface using the 
Volume of Fluid method: the location of the interface is represented by the volume fraction $C_{i j}$ of fluid 1 in the computational cell $i, j$ [19 21, 16 We have $0<C_{i j}<1$ in cells cut by the interface and $C_{i j}=0$ or 1 away from it. The propagation of the interface at velocity $U$ is performed in several steps. In a first step, the interface is reconstructed in each cell independently. Linear segments of slope approaching that of the interface are constructed, in the so-called Piecewise Linear Interface Construction (PLIC). The construction uses a local interface normal $\mathbf{n}$ estimated using an 8 point (in 2D) centered finite difference of $C_{i j}$. In a second step, the interface motion is calculated in a Lagrangian manner with velocities obtained by linear interpolation. Finally the volume fractions are recalculated. With the PLIC method [19 21, 16], the position of the interface is reconstructed with errors of order $\mathcal{O}\left(\kappa h^{2}\right)$, where $\kappa$ is the local curvature, for the position of the interfaces, and thus more accurately than in most volume fraction methods, including that of ref. 8]. During the simulations, we observe that only a very small fraction of the mass is lost. In the complex case of Fig. 3 this fraction is less than $210^{-3}$ over the entire simulation.

We performed our simulations in a square periodic box of size $5 D \times 5 D$. The simulation was initialized with a uniform velocity $U$ in the gas and the liquid droplet at rest. In all simulations reported here we kept $R e_{L}=2,000$, the liquid to gas density ratio $\rho_{L} / \rho_{G}=10$ and $R e_{G}=1,000$. Several droplet deformation and break up scenarios have been unveiled for varying $W e_{G}$. The first scenario is shown on Fig. 1. As a result of the presence of two rear-vortices engendered by the droplet motion, a concavity develops in the droplet surface which takes a typical "banana-shaped" configuration, with its concave side facing downstream (Fig 1(b) and Fig. 2). These vortices further stretch the droplet until rupture occurs near the tips. The coherent structures in the wake of the droplet are characteristic of 2D turbulence. A boundary layer develops on the front of the droplet as predicted by Taylor [15]. However at this $W e_{G}$ the boundary layer is stable.

The "mother" droplet may again break in a similar fashion thus generating additional children droplets. Alternatively, if $W e_{G}$ is sufficiently high second-generation droplets may also break up, producing third-generation droplets in a kind of bifurcation cascade. Moderate 
resolution $\left(256^{2}\right)$ and high resolution $\left(512^{2}\right)$ simulations exhibited similar results.

Simulations at larger Weber numbers such as the $W e_{G}=100$ simulation of Fig. 3 show the formation of much smaller scale structures. The droplet forms elongated filaments. The boundary layer on the front side of the droplet is now unstable, and horn-like structures typical of the Kelvin-Helmholtz instability are seen to grow while they are transported downstream (see Fig. 3(b)).

Yet another mechanism is shown on Fig. 4. There the droplet elongates then makes a bag with its concave side facing downstream. The bag closes, then breaks on the upstream side before yielding several separated droplets.

The simulation, although 2D, may be qualitatively compared to the experimental results. There are qualitative similarities, such as the breaking near the tips on Fig. 1)(b) or the bag formation. The $W e_{G}=100$ simulation shows the sheet stripping mode mechanism reported by [12] for $100<W e_{G}<350$. The most important difference occurs at $W e_{G}=10$ where in experiments the concave side faces mostly upstream. We believe that this difference arises because of the initial conditions we use, which result in a jump of the velocity - a vortex sheet - at the interface. This vortex sheet rolls up behind the droplet and creates structure seen on Fig. 2). Work is in progress to investigate the influence of initial conditions.

In conclusion, the results highlight the power of advanced numerical techniques to unveil the fascinating complexity resulting from the nonlinear interplay between gas-liquid interface and gas vortex motion. In particular, the crucial role played by coherent vortical structures suggests that the inclusion of the $R e_{G}$ dependence is key to the formulation of more advanced and realistic break up criteria. On the other hand, these results also indicate that caution is needed before the results provided by the numerical tool can be effectively converted into quantitative information of engineering interest, such as phase-diagrams and similar data.

One difficulty rests with the slow convergence of such calculations with the number of grid-points. In some regimes, the lower resolution experiments produce a similar picture as provided by higher-resolution ones, the main difference being that transitions in phasediagrams occur at different $W e_{G}$. In some instances, however, genuinely new mechanisms 
arise: the bag mechanism of Fig. U was observed only in $512^{2}$ simulations. A second difficulty is the two-dimensional nature of these calculations. As is well known, coherent vortical structures behave quite differently in two with respect to three-dimensions, and so should break up mechanisms. The Rayleigh Instability, which plays such an important role in the final stages of capillary driven break up [5], is absent in 2D. (However this may affect only the smallest scales of a large $W e$ break up). Despite these difficulties, we regard two-dimensional simulations as a very useful warm-up for more realistic three-dimensional investigations. While the former are already rather computationally expensive (about 3 CPU seconds per time step for a $512^{2}$ resolution on a IBM RS/6000 mod. 590 workstation), the latter set a pressing demand for high-resolution computations requiring the use of the most powerful present-day supercomputers. 


\section{FIGURES}
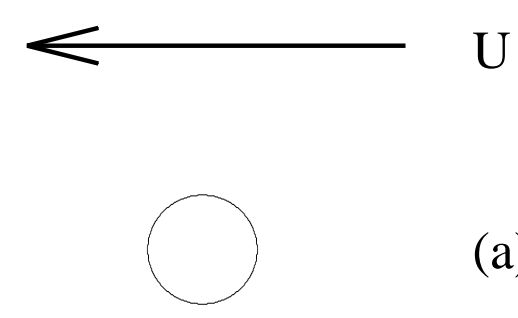

(a)

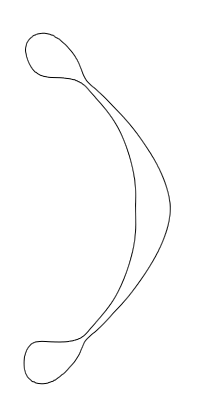

(b)

(c)

FIG. 1. Simulations of droplet break up for $W e_{G}=10.512^{2}$ grid points in a periodix box are used. 


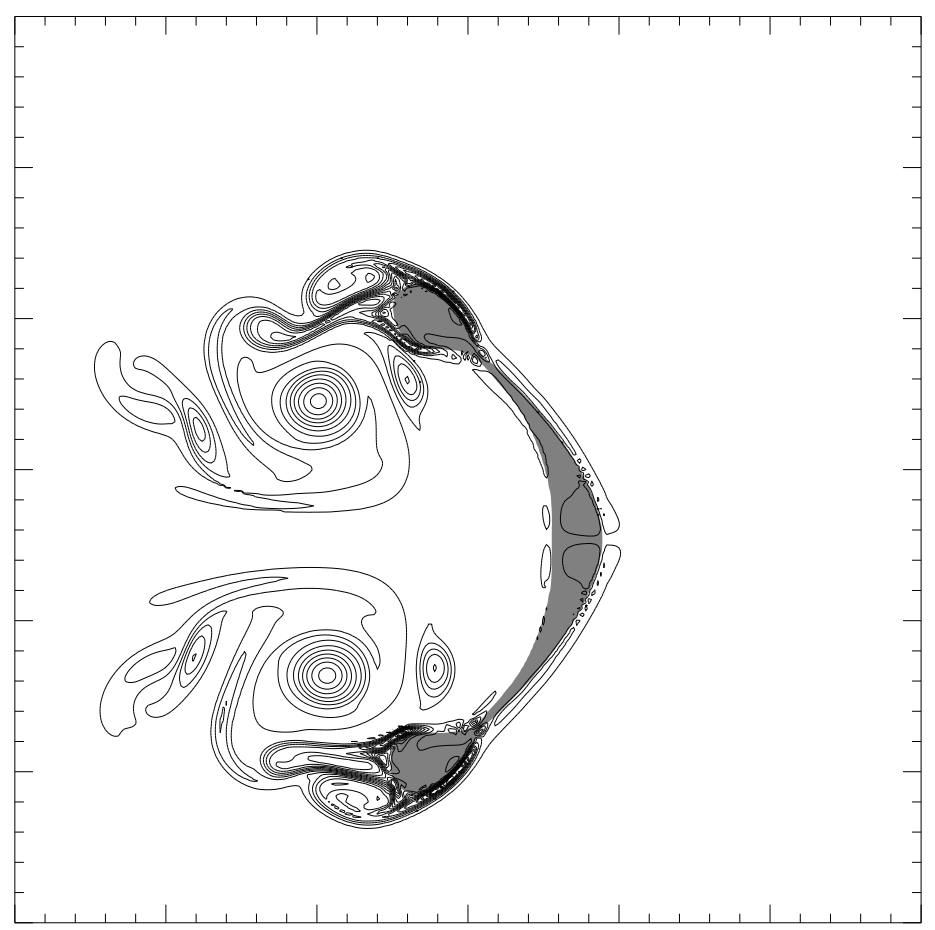

FIG. 2. Same as figure 1b, but with vorticity contours shown. 


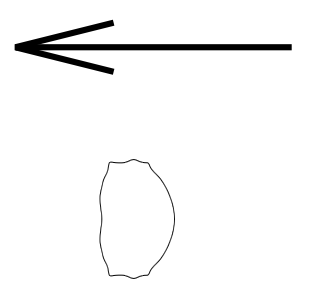

(a)

(b)

(c)

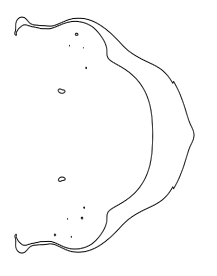

(d)

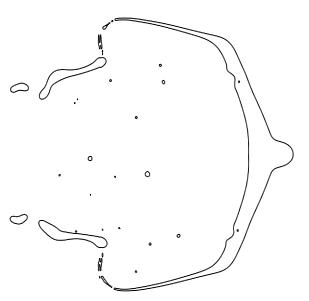

(e)

FIG. 3. Simulations of droplet break up for $W e_{G}=100$. 


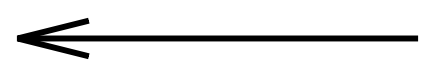

U

(a)

(b)

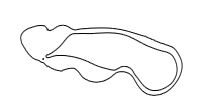

(d)

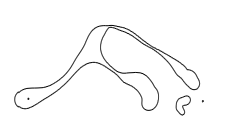

(e)

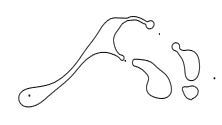

(f)

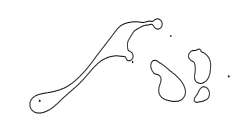

(g)

FIG. 4. Simulations of droplet break up for $W e_{G}=20$. 


\section{ACKNOWLEDGEMENTS}

DRET, CNES and SEP are gratefully acknowledged for their funding. The authors wish

to thank Daniel Lhuillier for illuminating discussions. S. Succi wishes to thank LMM for their kind hospitality and financial support. 


\section{REFERENCES}

* Present Address: IBM European Center for Scientific and Engineering Computing, Piazzale Giulio Pastore, 7, 00144 Roma, Italy.

[1] S. Tannever and G. Vasconcelos, Phys. Rev. Lett. 73, 2845 (1994).

[2] R. Goldstein, A. Pesci, and M. Shelley, Phys. Rev. Lett. 70, 343 (1993).

[3] P. Constantin et al., Phys. Rev. E 47, 4169 (1993).

[4] H. A. Stone, Annual Reviews of Fluid Mechanics 26, 65 (1994).

[5] X. D. Shi, M. P. Brenner, and S. R. Nagel, Science 265, 157 (1994).

[6] M. Brenner, X. Shi, and S. Nagel, Phys. Rev. Lett. 73, 3391 (1994).

[7] G. Batchelor, An Introduction to Fluid Dynamics, (Cambridge University Press, Cambridge, UK, 1967).

[8] B. Lafaurie et al., J. Comp. Phys. 113, 134 (1994).

[9] A. Wierzba, Experiments in Fluids 9, 59 (1990).

[10] S. A. Krzeczkowski, Int. J. Multiphase Flow 6, 227 (1980).

[11] P. G. Simpkins, Nature Physical Science 233, 31 (1971).

[12] M. Pilch and C. A. Erdman, Int. J. Multiphase Flow 13, 741 (1987).

[13] J. Kitscha and G. Kocamustafaogullari, Int. J. Multiphase Flow 15, 573 (1989).

[14] A. B. Liu and R. D. Reitz, Atomization and Sprays 3, 55 (1993).

[15] G. I. Taylor, in The Scientific Papers of G.I. Taylor, vol. III (Cambridge University Press, Cambridge, UK, 1963).

[16] J. Li, C. R. Acad. Sci. Paris, série IIb, (Paris) 320, 391 (1995).

[17] A. J. Chorin, Mathematics of Computing 22, 745 (1968). 
[18] W. H. Press and S. A. Teukolsky, Computers in Physics SEP/OCT, 514 (1991).

[19] D. Youngs, in Numerical methods for fluid dynamics, edited by K. M. Morton and M. J. Baines (Academic Press, New York, 1982), pp. 27-39, Institute for Mathematics and its Applications.

[20] E. G. Puckett and J. S. Saltzman, Physica D 60, 84 (1992).

[21] J. Y. Poo and N. Ashgriz, J. Comp. Phys. 93, 449 (1991). 